\title{
Clinical evidence for a biological effect of epigenetically active decitabine in relapsed or progressive rhabdoid tumors
}

\author{
Mona Steinbügl $^{1} \quad \mid$ Karolina Nemes $^{1} \quad \mid$ Pascal Johann $^{1,16}$ | Thomas Kröncke ${ }^{2}$ \\ Stefanie Tüchert $^{2}$ | Maria Joao Gil da Costa ${ }^{3}$ | Martin Ebinger ${ }^{4}$ (1) | Ulrich Schüller ${ }^{5,6,7}$ \\ Astrid Sehested $^{8}$ ｜ Peter Hauser ${ }^{9}$ [ | Harald Reinhard ${ }^{10}$ ｜ David Sumerauer ${ }^{11}$ | \\ Simone Hettmer ${ }^{12}$ (c) | Marcus Jakob ${ }^{13}$ ｜ Martin Hasselblatt ${ }^{14}$ ｜ Reiner Siebert ${ }^{15}$ | \\ Olaf Witt $^{16} \quad$ Joachim Gerss ${ }^{17} \quad$ Kornelius Kerl ${ }^{18}$ [ $\quad$ Michael C. Frühwald ${ }^{1}$ (c) \\ ${ }^{1}$ University Medical Center Augsburg, Paediatric and Adolescent Medicine, Swabian Children's Cancer Center, Augsburg, Germany \\ ${ }^{2}$ Department of Diagnostic and Interventional Radiology, University Medical Center, Augsburg, Germany \\ ${ }^{3}$ Pediatric Hematology and Oncology Division, University Hospital S. João Alameda Hernani Monteiro, Porto, Portugal \\ ${ }^{4}$ Department of General Pediatrics, Hematology and Oncology, Children's University Hospital, Tübingen, Germany \\ ${ }^{5}$ Department of Pediatric Hematology and Oncology, University Medical Center Hamburg-Eppendorf, Hamburg, Germany \\ ${ }^{6}$ Research Institute Children's Cancer Center Hamburg, Hamburg, Germany \\ ${ }^{7}$ Institute of Neuropathology, University Medical Center Hamburg-Eppendorf, Hamburg, Germany \\ ${ }^{8}$ Department of Paediatrics and Adolescent Medicine Rigshospitalet, Copenhagen, Denmark \\ ${ }^{9}$ Department of Pediatric Oncology, 2nd Department of Pediatrics, Semmelweis University, Budapest, Hungary \\ ${ }^{10}$ Department of PediatricsAsklepios Kinderklinik Sankt Augustin, Sankt Augustin, Germany \\ ${ }^{11}$ Department of Pediatric Hematology and Oncology, University Hospital Motol, Prague, Czech Republic \\ 12 Division of Pediatric Hematology and Oncology, Department of Pediatric and Adolescent Medicine, University Medical Center Freiburg, University of Freiburg, \\ Freiburg, Germany \\ ${ }^{13}$ Department of Pediatric Hematology, Oncology and Stem Cell Transplantation, University Hospital of Regensburg, Regensburg, Germany \\ ${ }^{14}$ Institute of Neuropathology, University Hospital Münster, Münster, Germany \\ ${ }^{15}$ Institute of Human Genetics, University of Ulm and Ulm University Hospital, Ulm, Germany \\ ${ }^{16}$ Hopp Children's Cancer Center Heidelberg (KiTZ), German Cancer Research Center (DKFZ) and Heidelberg University Hospital, Heidelberg, Germany \\ 17 Institute of Biostatistics and Clinical Research, University of Münster, Muenster, Germany \\ ${ }^{18}$ Department of Pediatric Hematology and Oncology, University Children's Hospital Münster, Münster, Germany
}

\section{Correspondence}

Michael C. Frühwald, University Children's Hospital Augsburg, Swabian Children's Cancer Center, Stenglinstraße 21, 86156 Augsburg, Germany.

Email:michael.fruehwald@uk-augsburg.de

\begin{abstract}
Background: Refined therapy has helped to improve survival rates in rhabdoid tumors (RT). Prognosis for patients with chemoresistant, recurrent, or progressive RT remains dismal. Although decitabine, an epigenetically active agent, has mainly been evaluated
\end{abstract}

Abbreviations: AML, acute myeloid leukemia; ATRT, atypical teratoid rhabdoid tumor; BSA, body surface area; BW, body weight; CR, complete remission; CTC, common toxicity criteria; eMRT, extracranial malignant rhabdoid tumor; GLM, germline mutation; HDCT, high-dose chemotherapy; HR, hazard ratio; MRT, malignant rhabdoid tumor; OS, overall survival; RT, rhabdoid tumor; RTK, rhabdoid tumor of the kidney; SD, stable disease; TTP, time to progression. 
in the management of hematologic malignancies in adults, safety in children has also been demonstrated repeatedly.

Materials and methods: A retrospective series of patients who received decitabine upon relapse or progression following therapy according to the EU-RHAB regimen is presented. Due to the retrospective nature of analyses, response was defined as measurable regression of at least one lesion on imaging. 850k methylation profiling was done whenever tumor tissue was available.

Results: A total of 22 patients with RT of any anatomical localization were included. Most patients (19/22) presented with metastases. All received low-dose decitabine with or preceding conventional chemotherapy. Patients received a median of two (1-6) courses of decitabine; $27.3 \%$ (6/22) demonstrated a radiological response. Molecular analyses revealed increased methylation levels in tumors from responders. No excessive toxicity was observed. Clinical benefits for responders included eligibility for early phase trials or local therapy. Responders showed prolonged time to progression and overall survival. Due to small sample size, statistical correction for survivorship bias demonstrated no significant effect on survival for responders.

Conclusions: Patients with RT demonstrate promising signs of antitumor activity after multiagent relapse therapy including decitabine. Analyses of methylation data suggest a specific effect on an epigenetic level. We propose to consider decitabine and other epigenetic drugs as candidates for further clinical investigations in RT.

KEYWORDS

ATRT, decitabine, malignant rhabdoid tumor, relapsed and refractory rhabdoid tumors

\section{1 | INTRODUCTION}

Rhabdoid tumors (RT) are aggressive malignancies affecting very young children. The entity comprises tumors of the central nervous system (ATRT) as well as extracranial malignant rhabdoid tumors (eMRT) of soft-tissue and rhabdoid tumors of the kidney (RTK). RT are characterized by genomic alterations in SMARCB1 or rarely, SMARCA4; $25 \%-35 \%$ of all patients carry a germ line mutation (GLM). ${ }^{1-4}$ Apart from surgical resection and conventional chemotherapy, radiotherapy, and high-dose chemotherapy (HDCT) have been established as elements of multimodal therapy. ${ }^{5-9}$ For patients nonresponsive to first-line therapy or who experience relapse, options are scarce and prognosis is dismal. In a recent analysis of 100 patients with eMRT, only $11.1 \%$ of patients with refractory or chemoresistant disease (analyzed within four months from diagnosis), and $26.6 \%$ with early relapse (analyzed at 12 months from diagnosis) survived five years following diagnosis. ${ }^{10}$ Long-term survival in patients with refractory or relapsed ATRT was only $14 \%$ and $5 \%$, respectively, in a cohort of 143 patients. ${ }^{11}$ Analysis of large ATRT and eMRT collectives recently discovered significant differences on an epigenetic level. ${ }^{12-15}$ Of the three subgroups of ATRT reported by Johann et al., two (ATRTTYR and - $\mathrm{SHH}$ ) demonstrate global hypermethylation. Consistently, we sought to test the demethylating ability and potential clinical benefit of low-dose decitabine (maximum of $20 \mathrm{mg}$ per $\mathrm{m}^{2}$ body surface area or $0.7 \mathrm{mg}$ per $\mathrm{kg}$ bodyweight per day) for relapsed or refractory RT.

Decitabine, originally developed as a cytotoxic agent, exerts epigenetic effects especially at lower doses and has activity in the therapy of malignancies with known hypermethylated genomes (e.g., myelodysplastic syndrome, acute myeloid leukemia (AML)). ${ }^{16,17}$ Safe use of the drug in children also in combination with conventional cytostatics has repeatedly been documented. ${ }^{18-21}$ Decitabine has been proven to penetrate the blood-brain barrier. ${ }^{22}$

Encouraged by these data, decitabine was used in individual treatment attempts for relapsed or refractory RT. Here we report a case series of such patients and evaluate possible clinical benefits of decitabine as an adjunct to conventional chemotherapy.

\section{MATERIALS AND METHODS}

\section{1 | Patients}

We included patients enrolled into the EU-RHAB registry who demonstrated relapse or progression on therapy and who had received at least one course of decitabine. Patients were from the Czech Republic, Denmark, Germany, Hungary, and Portugal. All patients had been treated according to the EU-RHAB consensus recommendations 
(www.rhabdoid.de). ${ }^{23}$ EU-RHAB is an international registry for RT of all anatomical locations, collecting data from primary and relapsed or refractory cases. ${ }^{23}$ To better understand individual approaches toward relapsed or refractory patients, we analyzed individual strategies for affected patients (December 2015 to March 2019). Two eligible patients were excluded due to incomplete data. Diagnoses had been confirmed by INI-1 negativity (proving SMARCB1-deficiency) employing WHO criteria in all patients. We did not observe any SMARCA4-negative case. We retrieved basic clinical and treatment information from the EU-RHAB database. Additional data were retrospectively collected from treating institutions by case report forms. Missing data were obtained by personal contact with treating institutions (mail and structured telephone interviews).

\subsection{Ethical considerations}

The EU-RHAB consortium has received continuous ethical approval for more than 10 years (Registry: ID 2009-532-f-S, latest amendment 12/2016; Relapse/Progression: ID 2018-302-f-S). The decision for an off-label attempt of decitabine was made by the treating physicians at the respective institutions following expert counseling by the principal investigator of the EU-RHAB registry. Recommendations always prioritized participation in clinical trials, whenever available. It also considered the results of analyses from molecular tumor boards such as INFORM. ${ }^{24}$ Patients and/or legal guardians (depending on age) provided informed consent following disclosure of the experimental nature of treatment and information on potential risks and benefits. The counseling process as such and collection of pertinent data were approved by the ethics committee of the LMU München, Project-Nr. 19-269.

\subsection{Assessments}

Clinical assessments and time points for imaging did not follow a standardized protocol as patients were treated individually and at different time points in relation to other therapeutic elements. Toxicity was either reported according to common toxicity criteria (CTC) or extracted from written reports. For statistical purposes, patients were categorized as having experienced "relevant toxicity" or not, with relevant being defined as grade 4 toxicity according to CTC or any other event unexpected or requiring prolonged hospitalization.

Primary distinction into responders and non-responders was based on imaging and accompanying documentation of the clinical course provided by the treating institution. All imaging was evaluated by local radiologists followed by review at the reference radiology institution of the EU-RHAB consortium (Department of Diagnostic and Interventional Radiology, University Medical Center Augsburg). Response to therapy was defined as objective, measurable regression of at least one lesion on magnetic resonance $(n=21)$ or computer tomography $(n=1)$ imaging. Response status was not necessarily concordant with the response assessment as defined in the EU-RHAB protocol. Stan- dardized criteria for response assessment such as RANO or RECIST could unfortunately not be applied due to a lack of uniform imaging protocols in the retrospective data set and the exploratory approach of this work.

\subsection{Statistical analysis}

IBM SPSS Statistics for Windows, version 24.0 and SAS, version 9.4 for Windows were used for survival analysis. Survival was measured from the first day of decitabine treatment. Kaplan-Meier analyses were made for time to progression (TTP) and overall survival (OS). The effect of decitabine treatment on TTP and OS was evaluated by Cox regression with a time-dependent response indicator. Results were deemed significant at $p \leq 0.05$. For the statistical assessment of responders among the molecular subgroups, a chi-square test was performed ( $R$, v.3.6.2).

\section{5 | Designation of molecular subgroups}

Data on methylation profiles of tumors were either analyzed by $850 \mathrm{k}$ methylation arrays or IDATs from previous analyses were retrieved. To allocate tumors to specific DNA methylation subgroups, we used the most recent version of the Heidelberg methylation array classifier. ${ }^{12,25}$ In addition, tSNE analysis was performed as an orthogonal validation of the random-forest-based classification results to confirm subgroup allocations. As eMRT almost exclusively cluster with the ATRTMYC subgroup, patients with eMRT were excluded from subgroup allocation

\section{3 | RESULTS}

A total of 22 patients were included. Rescue modalities were applied according to individual circumstances, and included surgery, high-dose chemotherapy, and radiotherapy. Demographics, disease and treatment characteristics are summarized in Table 1. Following first-line therapy, 10/22 (45.5\%) children had achieved a complete remission but subsequently relapsed; $n=12 / 22$ (54.5\%) did not achieve a complete remission and were in progression at the time of decision for salvage treatment. Different individual treatment concepts were initiated depending on clinical variables and availability of molecular information. Localized lesions were submitted to surgery or radiotherapy in 4/22. In 2/22 other innovative treatment regimens ( $n=1$ metronomic therapy according to MEMMAT, $n=1$ paclitaxel, carboplatin and melphalan) were initiated, but either therapy was stopped due to rapid progression. For the remaining 16 patients, use of decitabine was the first treatment approach deviating from conventional chemotherapy approaches such as the EU-RHAB regimen. Decitabine was administered preceding conventional chemotherapy (Figure 1a). An example of an often-used standard treatment regimen is provided in Figure $1 \mathrm{~b}$. In specific cases, duration or doses were adjusted individually (for further details, see Supporting Information Table S1). 
TAB LE 1 Patient characteristics and prior treatment

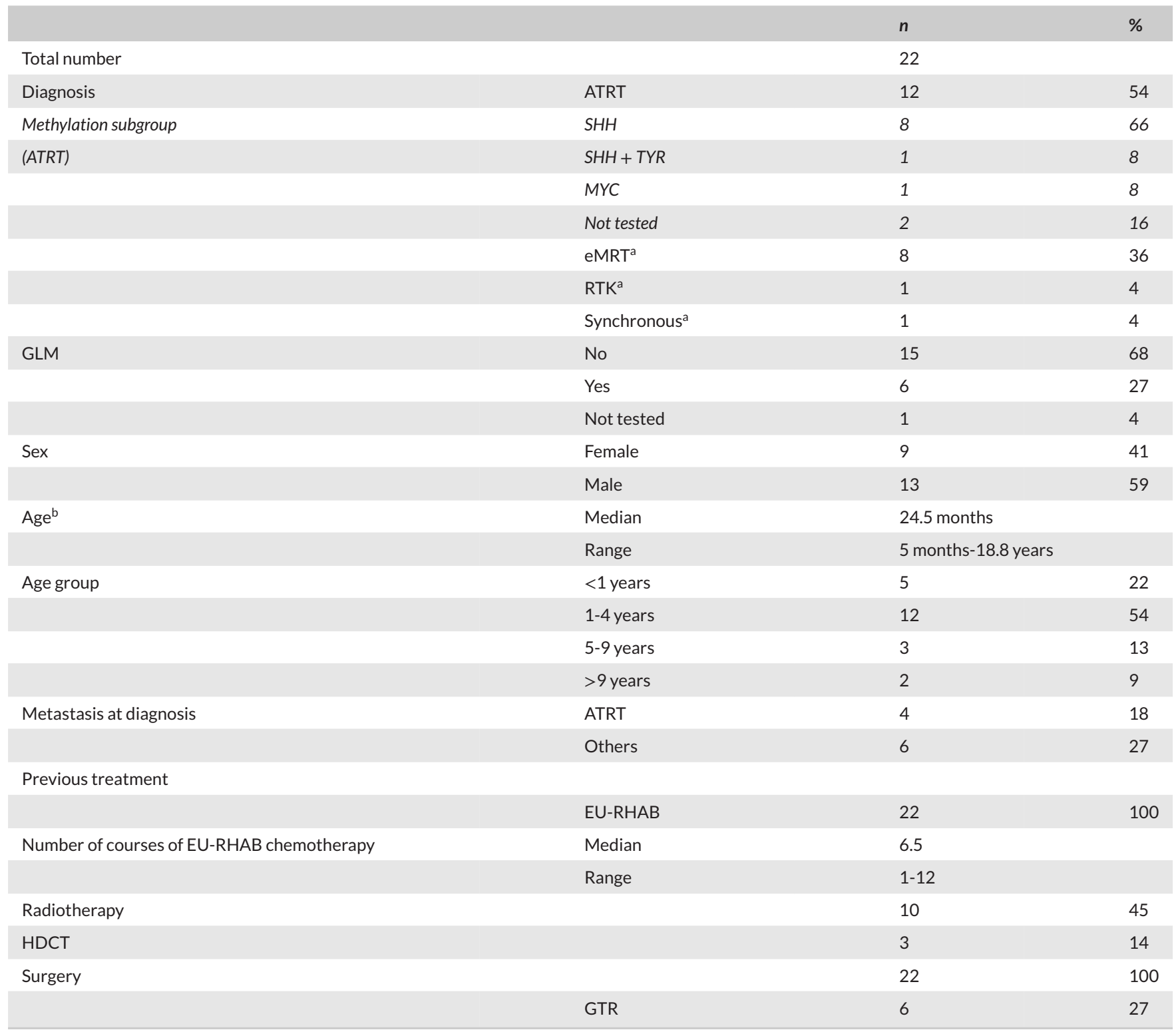

Abbreviations: ATRT, atypical teratoid rhabdoid tumor; eMRT, extracranial malignant rhabdoid tumor; GLM, germ line mutation; M+, tumor dissemination (solid metastasis, meningeosis, tumor cells in liquor); $M-$, no dissemination; RTK, rhabdoid tumor of the kidney.

a Methylation data available for two eMRT, one RTK, and one RTK in patient with synchronous tumor. All clustered with MYC.

${ }^{\mathrm{b}}$ Age at first decitabine treatment.

\subsection{Decitabine elicits antitumor activity in patients with relapsed and progressive RT}

To assess the effects of therapy enhancement employing decitabine, the proportion of patients who showed signs of antitumor activity was analyzed. In 6/22 (27.3\%), a radiological response as defined above was noted. Of the remaining patients, 4/22 (18.2\%) did not demonstrate any evaluable lesions at the time of decitabine treatment due to prior local treatment and $n=12 / 22$ (54.5\%) revealed no response. Notably, among non-responders, one patient presented with stable disease and in two more patients a clinical response was reported, which was not visible on imaging (improvement in neurological status in an ATRT or regression of palpable mass in a superficial soft-tissue eMRT).
Characteristics of responders in comparison to non-responders and those with non-evaluable disease are listed in Table 2. Responses included decrease in primary tumor size and regression of solid metastases or meningeosis (see Figure 2 for exemplary imaging and Table 3 for characterization of the radiological findings). Patients received decitabine until scheduled completion $(n=4)$, progression on therapy $(n=15)$, or when excessive hematologic toxicity occurred $(n=1)$. One patient was moved to local therapy and subsequently enrolled into a phase I trial (NCT02601937) without signs of progression (patient 7 , see case reports), one patient stopped treatment due to parental decision.

Following treatment with decitabine, $8 / 22$ patients received no further tumor-directed therapy due to progression or death. Four patients 
TAB LE 2 Characteristics of responders, non-responders, and patients not evaluable for a response

\begin{tabular}{|c|c|c|c|c|c|c|c|}
\hline & & \multicolumn{2}{|c|}{ Responder } & \multicolumn{2}{|c|}{ Non-responder } & \multicolumn{2}{|c|}{ Not evaluable } \\
\hline & & $n$ & $\%$ & $n$ & $\%$ & $n$ & $\%$ \\
\hline Total number & & 6 & 100 & 12 & 100 & 4 & 100 \\
\hline & ATRT & 4 & 67 & 7 & 58 & 1 & 25 \\
\hline & eMRT & 2 & 33 & 3 & 25 & 3 & 75 \\
\hline & Synchronous & - & & 1 & 8 & - & \\
\hline \multicolumn{8}{|l|}{ GLM } \\
\hline & No & 4 & 67 & 7 & 58 & 4 & 100 \\
\hline & Yes & 2 & 33 & 4 & 33 & - & \\
\hline & $\mathrm{SHH}+\mathrm{TYR}$ & - & & 1 & 8 & - & \\
\hline & MYC & - & & 3 & 25 & 2 & 50 \\
\hline & Not tested & 2 & 33 & 5 & 42 & 1 & 25 \\
\hline \multicolumn{8}{|l|}{ Sex } \\
\hline & Male & 3 & 50 & 6 & 50 & 4 & 100 \\
\hline & Female & 3 & 50 & 6 & 50 & - & \\
\hline \multicolumn{8}{|l|}{ Age group } \\
\hline & $<1$ year & 1 & 17 & 4 & 33 & - & \\
\hline & 1-4 years & 4 & 67 & 6 & 50 & 2 & 50 \\
\hline
\end{tabular}

Abbreviations: ATRT, atypical teratoid rhabdoid tumor; eMRT, extracranial malignant rhabdoid tumor; GLM, germ line mutation; RTK, rhabdoid tumor of the kidney.

received palliative chemotherapy, 10 were subjected to another experimental therapy approach with a curative intent. Strategies included tazemetostat (NCT02601937) ( $n=3$ ), the RIST regimen, MEMMAT (NCT01356290 - no trial enrollment due to trial not open in country), metronomic chemotherapy according to Kieran et al., azacytidine, arsenic trioxide or TEMIRI. ${ }^{26-29}$ One patient underwent HDCT with subsequent autologous stem cell transplant. In addition, five patients received radiotherapy and one had surgery.

\subsection{Prolongation of TTP and EFS following decitabine}

All but one patient (95.5\%) had progressive disease at some point, and $19 / 22$ (86.4\%) have died. One patient, who was among the patients without evaluable lesions and received decitabine as part of adjuvant chemotherapy after local therapy, was alive in complete remission (CR) 24 months later. Two others are alive with disease: one was undergoing metronomic chemotherapy, and the other was in palliative care at most recent follow-up.

Median TTP for all patients was 7.6 weeks, and median OS for all patients 26.3 weeks. On Kaplan-Meier analyses, responders showed prolonged TTP and OS in comparison with non-responders (12 weeks vs. 3 weeks; 40 weeks vs. 15 weeks, respectively). Following statistical adjustment for survivorship bias, responses were not significant (TTP: $P \geq 0.05$; hazard ratio $(H R)=0.64$; OS: $P \geq 0.05$, HR $=1.11$ for comparison between responders and nonresponders). Patients without evaluable disease had a superior absolute TTP and OS (16 weeks; 45 weeks) but no significant survival benefit in comparison with responders (TTP: $P \geq 0.05 ; \mathrm{HR}=0.38$; OS: $P \geq 0.05$, $\mathrm{HR}=0.22$ ). 
TAB LE 3 Characteristics of radiological findings in responders

\begin{tabular}{|c|c|c|}
\hline Patient & Description of tumor & Description of response \\
\hline 7 & $\begin{array}{l}\text { ATRT with the primary tumor in the right frontal } \\
\text { lobe, multiple smaller metastases, mostly in } \\
\text { the cerebellum. } \\
\text { Metastatic lesions are of smooth, cystic } \\
\text { formation, isodense to cerebrospinal fluid, and } \\
\text { show no contrast agent uptake. }\end{array}$ & $\begin{array}{l}\text { Primary tumor same size as in the first study. } \\
\text { Size regression of the biggest metastatic lesion in the left } \\
\text { cerebellar hemisphere from } 8 \times 9 \times 8 \mathrm{~mm} \text { to } \\
4 \times 3 \times 4 \mathrm{~mm} \text {. MRI signal unchanged to previous } \\
\text { imaging. } \\
\text { On the left temporopolar region multiple, aggregated } \\
\text { smaller lesion with a total size of } 5 \times 6 \times 4 \mathrm{~mm} \text { on } \\
\text { previous imaging cannot be found in the second study. } \\
\text { Suspicious new lesion at the left tentorium, later identified } \\
\text { as new metastasis. } \\
\text { Due to the new lesion classified as progressive disease }\end{array}$ \\
\hline 10 & $\begin{array}{l}\text { Spinal relapse of ATRT with multiple } \\
\text { disseminated nodules. } \\
\text { Most prominent two nodules are the biggest one } \\
\text { at LWK } 2 / 3 \text { and a smaller one at BWK } 9 \text {. } \\
\text { Both are intraspinal, hypointense to } \\
\text { muscle/myelon, show contrast agent uptake } \\
\text { and have slightly irregular margins. }\end{array}$ & $\begin{array}{l}\text { Overall reduction of the intraspinal tumor mass. } \\
\text { The biggest metastatic nodule is not detectable in the } \\
\text { second study, but has been biopsied in between studies. } \\
\text { The smaller nodule, that due to the identical signaling is } \\
\text { definitely a metastasis, is also not detectable any more }\end{array}$ \\
\hline 11 & $\begin{array}{l}\text { Hepatic eMRT with peritoneal carcinosis and } \\
\text { lung metastasis. } \\
\text { Size measurement is difficult due to } \\
\text { dissemination. The whole liver is tumorus. In } \\
\text { the left hepatic lobe, an oval, polylobulated, } \\
\text { partly cystic, partly necrotic tumor nodule can } \\
\text { be circumscribed and measured. }\end{array}$ & $\begin{array}{l}\text { Mixed response with a measurable size reduction of the } \\
\text { primary tumor but further growth of metastasis. } \\
\text { Size regression of the circumscribable tumor nodule from } \\
4.2 \times 5.5 \text { to } 3.3 \times 3.5 \mathrm{~cm} \text {. Simultaneously definite size } \\
\text { increase of extra-hepatic tumor mass. } \\
\text { Due to the significant growth of the extra-hepatic tumor } \\
\text { mass classified as progressive disease }\end{array}$ \\
\hline 14 & $\begin{array}{l}\text { Leptomeningeal dissemination of ATRT with } \\
\text { T2-hypointense, noncontrast agent uptaking } \\
\text { lesions, that partially surround the myelon } \\
\text { along the whole spinal axis. }\end{array}$ & $\begin{array}{l}\text { Measurable regression of intraspinal metastasis along the } \\
\text { cervical and lumbosacral spinal canal. Exemplary is a } \\
\text { size decrease of tumor material in the sacral dura sac } \\
\text { from } 1.1 \times 0.4 \mathrm{~cm} \text { to } 0.3 \times 0.3 \mathrm{~cm} \text { (height } \times \text { depth) } \\
\text { Persistence of intracranial leptomeningeal dissemination }\end{array}$ \\
\hline 22 & $\begin{array}{l}\text { Infratentorial ATRT with progressive spinal } \\
\text { meningeosis. }\end{array}$ & Clear volume regression of spinal meningeosis $>50 \%$ \\
\hline
\end{tabular}

\subsection{Decitabine-enhanced therapy is well tolerated}

No treatment-related deaths were reported; one patient terminated treatment due to severe hematologic toxicity. A total of 10 patients (45.5\%) experienced 13 relevant toxicities, including hematologic toxicity $(n=6)$, as well as infections $(n=4)$, one case of mild veno-occlusive disease and one patient suffering from a disturbed sleep-wake cycle. One patient suffered from severe hyponatremia with respiratory insufficiency and seizures. This was attributed to administration of intraventricular methotrexate $(0.5 \mathrm{mg})$; a following course of decitabine was tolerated without significant toxicity.

\subsection{Subgroup distribution and methylation levels within the cohort}

For 9/12 patients with ATRT and 3/10 non-ATRT tumors, full data sets from $850 \mathrm{k}$ methylation profiling of the tumor prior to decitabine therapy were gathered. Apart from one case, all patients were unequivocally allocated to one subgroup, as demonstrated in the tSNE plot in Figure 3a. The average methylation level in tumors of patients with a radiological response to therapy was higher than in nonresponding patients (Figure $3 b$ ). With respect to subgroup, no significant correlation could be detected in ATRTs, most likely owing to small sample size and an overrepresentation of ATRT-SHH tumors (chi-square test statistics 0.375 ). For two of the patients (patient 14 and patient 17 , 
A

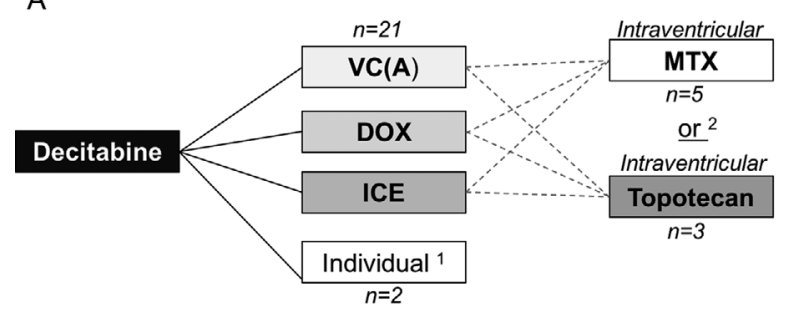

B

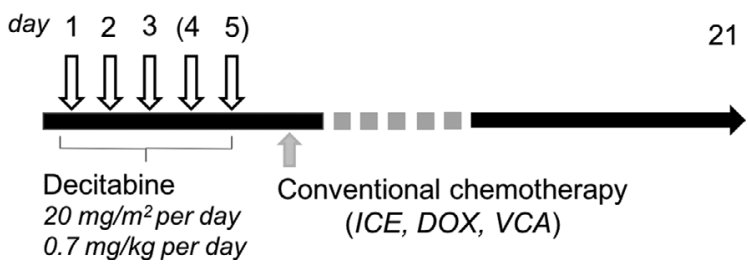

FIGURE 1 (a) Compound combinations. Decitabine was combined with different conventional chemotherapeutics. VC(A), DOX, ICE, and MTX were administered according to recommendations of the EU-RHAB registry, topotecan on an individual basis. VC(A), vincristine, cyclophosphamide (actinomycin D); DOX, doxorubicin; ICE, ifosfamide, carboplatinum, etoposide.; MTX, methotrexate. ${ }^{1}$ One patient received four courses of decitabine in combination with intraventricular topotecan only; one patient was given a combination of liposomal doxorubicin, melphalan, and decitabine. ${ }^{2}$ Intraventricular therapy was conducted in patients with central nervous system lesions if no contraindications were present, either with methotrexate or with topotecan. (b) Standard treatment regimen. Patients received at least two and up to five days of decitabine at the indicated doses. After completion of the decitabine prephase, conventional chemotherapy was started on the following day. Cycles restarted after day 21

both with ATRT-SHH) pre- and posttreatment samples were available. Comparison of average methylation levels found a difference of 0.011 (patient 14) and 0.014 (patient 17), thus showing indeed a decrease in overall methylation levels.

\subsection{1 | Case reports}

Patient 7 was diagnosed with a nonmetastatic ATRT-SHH. He achieved $\mathrm{CR}$ after resection, proton beam therapy, eight courses of conventional chemotherapy, and HDCT. He remained in remission for eight months until presenting with diffuse spinal dissemination, including two large solid spinal lesions. A diagnostic biopsy was taken, and molecular profiling through INFORM was initiated. Upon diagnosis of relapse, conventional chemotherapy was restarted, supported by the use of decitabine. At restaging following two decitabine-enhanced courses, metastases demonstrated a definitive size reduction. After an additional course, treatment was stopped to proceed to radiotherapy and the anticipated enrollment into a clinical trial (NCT02601937). A total of five months after start of decitabine and while on trial drug progress was noted; the patient died after four months of palliative care.

Patient 10 presented with ATRT-SHH, inoperable at diagnosis due to primary intracranial dissemination. He was treated with six courses of conventional chemotherapy according to EU-RHAB; a partial resection of the primary tumor was undertaken. At restaging, further progression of several lesions was seen, and individual therapy with escalation of conventional chemotherapy by adding decitabine was started. Regression of some solid lesions and meningeosis was seen after the first courses, but other tumor manifestations progressed at the same time. In palliative intention surgery and radiotherapy was administered for local control and palliative chemotherapy with temozolomide was initiated. The patient developed a secondary AML and succumbed shortly thereafter due to progressive disease (no GLM demonstrated).

Patient 16 experienced metastatic relapse to the lungs of a primarily nonmetastasized eMRT of the liver. CR had previously been maintained for 13 months after GTR, nine courses of conventional chemotherapy, radiotherapy, and subsequent maintenance therapy. As salvage treatment, metronomic chemotherapy (NCT01356290) was started, but rapid progression was noted on imaging shortly thereafter. Due to clinical deterioration, further treatment plans were terminated. The patient nonetheless stabilized, and treatment including decitabine was restarted, leading to a major clinical improvement and size regression of the pulmonary lesions. A total of four courses of decitabine were administered until further progression was noted. Due to the overall improved life expectancy, the patient was eligible for enrollment into a clinical trial (NCT02601937), remaining progression free for another eight weeks. The patient died of disease six months after the first dose of decitabine.

\section{4 | DISCUSSION}

To this day, diagnosis of RT is associated with a daunting prognosis. Despite intensive multimodal approaches, many patients suffer from early relapse or progression on therapy. ${ }^{11,23,30}$ Median OS in 99 patients from the EU-RHAB registry after relapse or progression on therapy was 18 weeks, and only $20 \%$ were alive one year after the event, emphasizing the desperate need for novel therapeutic approaches (Steinbügl et al., unpublished). In vitro studies have elucidated a multitude of affected pathways and mechanisms as a consequence of SMARCB1 loss in RT, unveiling new potential therapeutic targets. This includes among others the overexpression of Aurora Kinase $A,{ }^{31,32}$ upregulation of EZH2, ${ }^{33}$ as well as CDK4/CDK6/cyclin D1/RB pathway activation ${ }^{34,35}$ and many more. ${ }^{36}$

Translation of these findings into clinical trials has been challenging, mostly due to the low incidence and rapid course of disease. The number of clinical trials specifically aimed at patients with relapsed or refractory RT has been very limited, and clinical experiences have scarcely been published. Wetmore et al. reported a case series of four patients treated with the Aurora Kinase A inhibitor alisertib as a single agent in relapsed or refractory ATRT, where all patients displayed disease stabilization and/or regression. ${ }^{37} \mathrm{~A}$ subsequent clinical trial employing alisertib either as a single agent in relapsed or refractory RT or in combination with conventional chemotherapy in newly diagnosed RT is actively recruiting (NCT02114229). In a different trial, single-agent alisertib did not exhibit antitumor activity 

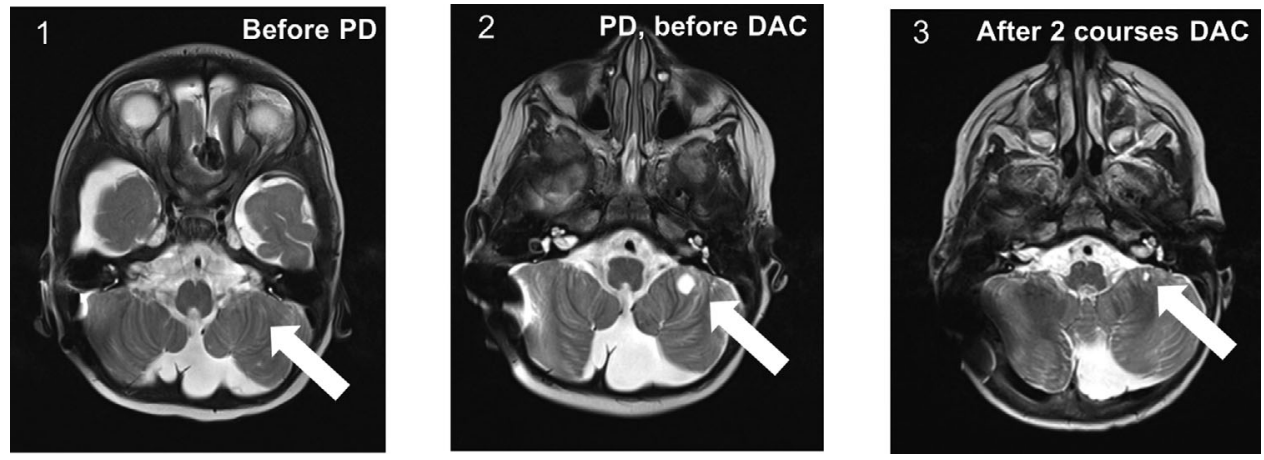

FIGURE 2 Exemplary imaging in a responder. MRI (axial T2 with contrast) of patient \#10 from 2 months before initiation of decitabine therapy and during first-line therapy, showing no cerebellar lesion (1); 3 weeks before initiation of decitabine therapy showing a new metastasic lesion in the cerebellum (2) and following 2 courses of decitabine plus conventional chemotherapy showing regression of the lesion (3). DAC, decitabine; $\mathrm{PD}$, progressive disease; MRI, magnetic resonance imaging

in four relapsed or refractory $\mathrm{RT}^{38}$ Gotti et al. and Berland et al. reported single cases of responses to metronomic chemotherapy regimens in ATRT using either vinorelbine, cyclophosphamide, and celecoxib or in the latter bevacizumab, liposomal cytarabine, celecoxib, cyclophosphamide, and etoposide..$^{39,40}$ In a phase I trial of the CDK4/6 inhibitor ribociclib, two of 15 patients with RT presented with stable disease (SD). The trial enrolled 32 patients with neuroblastoma, RT, rhabdomyosarcoma, and anaplastic meningioma, and best overall response was SD in nine patients. ${ }^{41} \mathrm{~A}$ phase I trial of the $\mathrm{EZH} 2$ inhibitor tazemetostat in SMARCB1-deficient tumors is ongoing (NCT02601937); responses have been reported in individual cases, and further results are pending. ${ }^{42}$

We analyzed 22 patients, who had received decitabine for therapy of tumor relapse or progression. To our knowledge, this is one of the largest, for the most part uniformly treated, cohorts of relapsed or progressive RT reported so far. Patients with intra- and extracranial RT had received a uniform first-line treatment according to EU-RHAB, minimizing the heterogeneity of the group. In this highly refractory setting, a remarkable $27.3 \%$ demonstrated radiological signs of antitumor activity following decitabine-augmented chemotherapy. We matched this observation with molecular data suggesting a correlation between response status and methylation signature. Median OS and TTP were prolonged in responders compared with non-responders, although not significantly.

Our provocative results are clearly limited by the individual treatment approaches outside of a clinical trial setting, the small cohort size, and the retrospective nature of the data. Robust survival analysis was accordingly limited. Furthermore, and as mentioned above, we chose a very broad definition of response on imaging, as we interpret size regression, however small, as a promising sign of antitumor activity. Imaging was conducted in individual treatment settings without standardized imaging protocols, precluding a systematic assessment according to standardized and validated criteria such as RANO or RECIST without having to exclude patients from this valuable collection of cases. The main objective of this work was an exploratory, clinical assessment of the potential biological activity of the agent. Naturally this reduces the comparability to similar relapse trials, as patients who were classified as responders may not have had a response as defined by RANO or RECIST criteria. Any confirmatory, prospective trials using decitabine will need to employ these validated criteria or even iRECIST, because it is unclear whether the established methods adequately assess novel therapeutic strategies, especially immunotherapy. ${ }^{43-46}$

As the sample size was expectedly too small for convincing statistical evaluation, we included case reports to illustrate real-life benefits after receiving decitabine-augmented salvage therapy. Case 10 explicitly demonstrates that primarily chemoresistant lesions were responsive to therapy enhancement with decitabine, hinting at a specific effect of the agent. Case reports 7 and 16 show that clinical benefits may include bridging to local therapy measures and enrollment into clinical trials as well as control of tumor-related symptoms. Early-phase trials typically demand a minimum life expectancy for enrollment; in case 16 this was made possible only through the temporary response seen after addition of decitabine to the therapy regimen.

Decitabine is an FDA- and EMA-approved agent, which has passed multiple safety trials alone and in combination with chemotherapy in children and adults. ${ }^{16,18,47,48}$ We also observed the frequently described hematologic toxicity; however, no toxic death occurred and only one patient was taken off medication due to side effects. In a cohort of 143 ATRT patients treated with an unaltered EU-RHAB regimen, the majority of patients demonstrated grade 3-4 hematologic toxicity, allowing the conclusion that adding decitabine did not lead to disproportionate added toxicity. ${ }^{11}$

The rationale for the use of decitabine in RT is its potential for demethylation. As proof of principle, we demonstrate that pre- and posttreatment tumor samples in two patients did indeed have lowered methylation levels. Thus, we included methylation profiling data and methylation subgroup allocation into our analyses. The majority of ATRTs matched to the SHH subgroup. This is consistent with recently published data demonstrating $\mathrm{SHH}$ as a negative predictive factor of OS with an increased risk for metastatic disease, thus making these patients more likely to fail first-line therapy. ${ }^{11,49}$ Comparison of overall methylation levels suggests that differences in response to decitabineenhanced therapy might be influenced by tumor methylation levels. 
FIGURE 3 (a) Subgroup allocation. tSNE plot of the samples for which methylation profiling is available $(n=12)$. In this subcohort, a total of seven patients were allocated to the ATRT-SHH subgroup, one patient to ATRT-MYC and for one patient, discrepant subgrouping between two distinct tumor samples revealed ATRT-TYR and ATRT-SHH. The patients with non-ATRT tumors were considered separately as subgroup "eMRT." For this image, only the primary tumors were considered. Tumors that responded to therapy are highlighted in green, nonresponding patients in orange. As a reference cohort, 387 ATRT and eMRT were derived from Ho et al. ${ }^{72}$

(b) Methylation levels. Indicates average methylation levels per sample (y-axis). The patients receiving decitabine have been grouped into responders $(n=3)$ or nonresponders $(n=8)$. As a reference, the ATRT subgroups and the eMRT subgroup are depicted as well a)

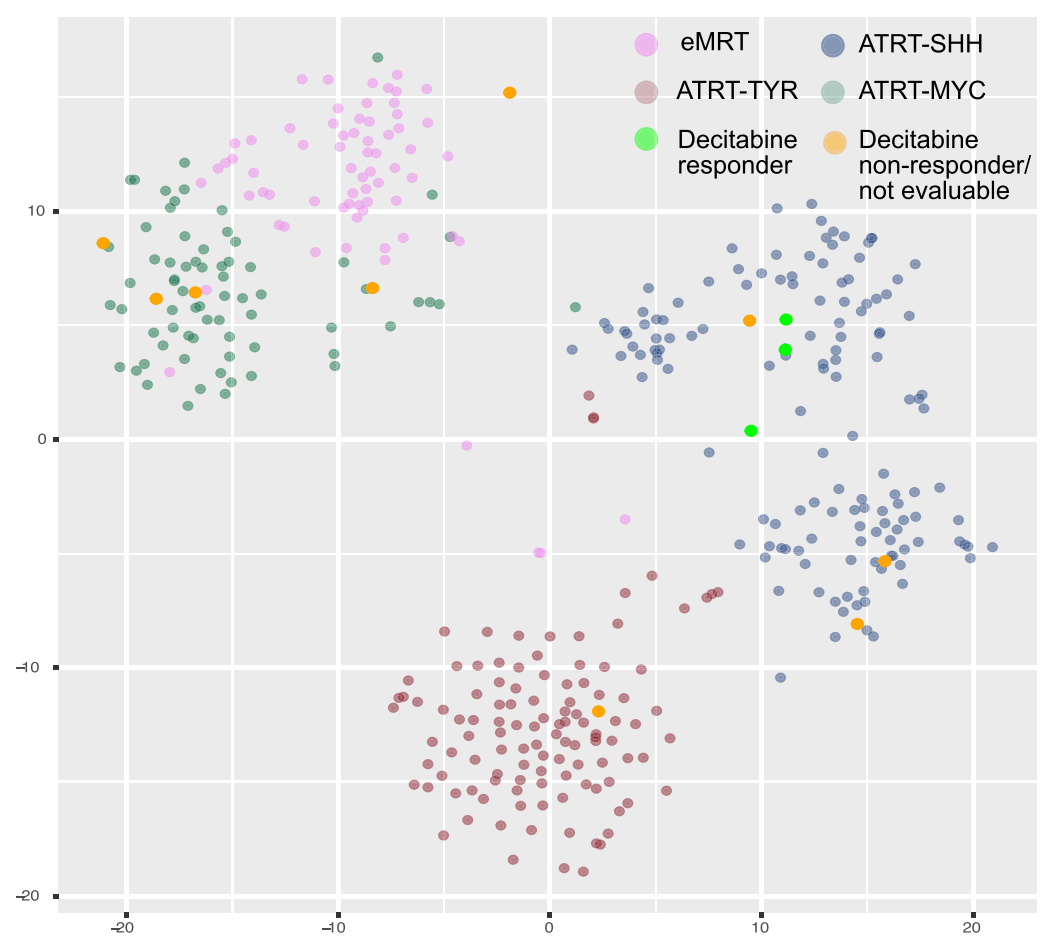

b)

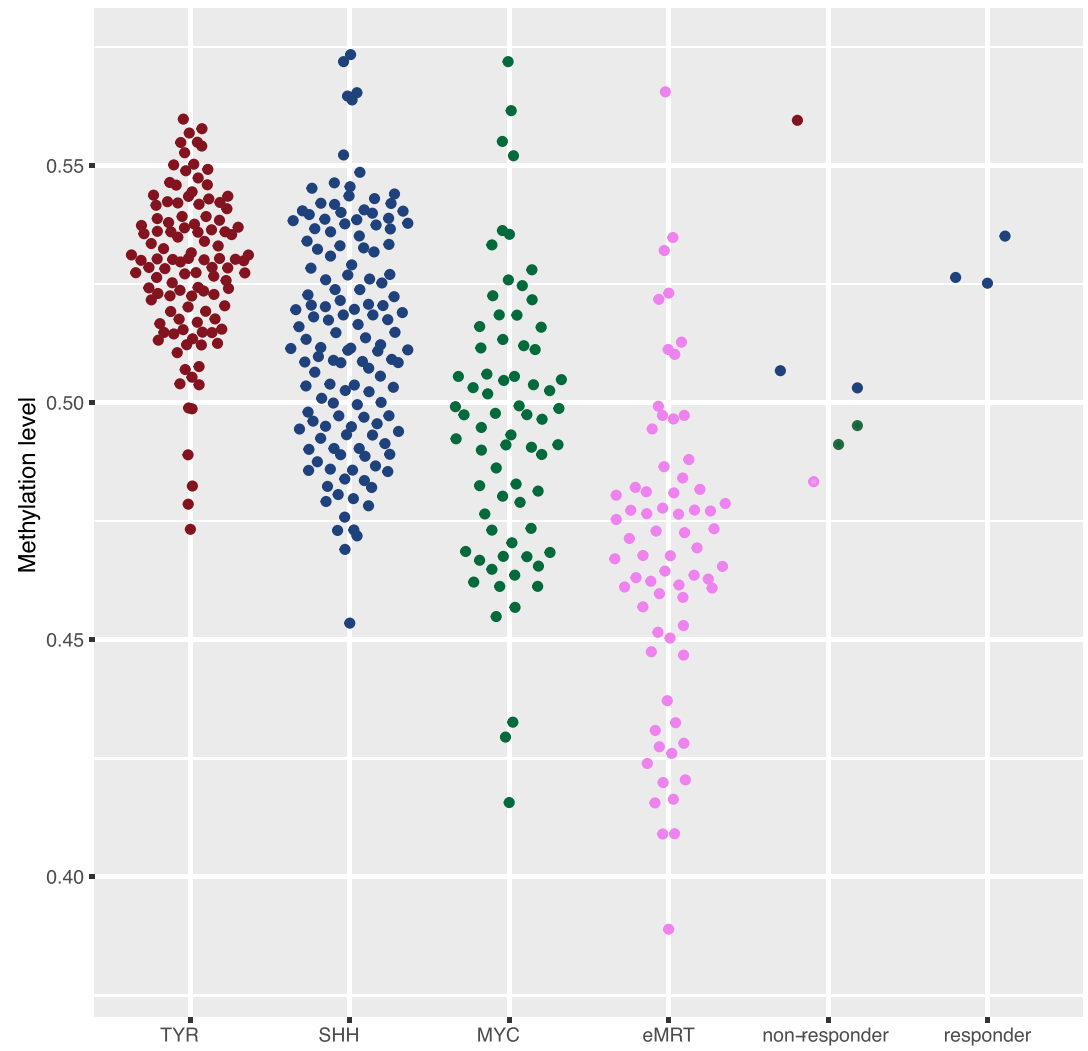

Consistently, future investigations should review methylation levels as a potential marker for targeted therapy with demethylating agents in RT.

In addition, it has been postulated that the overall antitumor effect of decitabine is facilitated by resensitization of tumor cells to chemotherapy, synergistic effects with platin-based agents, and immunomodulation. ${ }^{50-67}$ Recent findings suggested high immunogenicity of RT. ${ }^{68,69}$ It seems logical that future clinical use of decitabine should be in combination with immune-checkpoint inhibitors or other agents targeting the immunogenicity of MRT; an early phase I trial has already been rolled out. ${ }^{70}$ Other epigenetically active agents, such as decitabine's close structural analogue, azacytidine, should presumably 
be included in preclinical and clinical evaluation of the use of these agents in RT. ${ }^{71}$

Despite the dismal prognosis of relapsed and refractory MRT, there is a lack of access to controlled clinical trials with innovative agents targeting specific molecular characteristics. This has led to repeated individual treatment attempts, using decitabine for enhancement of conventional chemotherapy. In the current cohort, we detected indicators of antitumor activity in a promising $27.3 \%$ of the cohort without severe safety concerns. We propose that decitabine could benefit patients with relapsed and refractory MRT and should be included into prospective clinical trials for these patients, preferably in a synergistic combination with further targeted agents.

\section{FUNDING}

EU-RHAB is supported by grants to MCF by the "Deutsche Kinderkrebsstiftung" DKKS 2010.03, the German Research Foundation (DFG) (FR 1516/4-1), and the parent organization "Lichtblicke."

\section{DATA AVAILABILITY STATEMENT}

The data that support the findings of this study are available in the supplementary material of this article or can be retrieved from the corresponding author upon reasonable request.

\section{ORCID}

Martin Ebinger (D) https://orcid.org/0000-0002-4229-8058

Peter Hauser (D) https://orcid.org/0000-0002-8307-8975

Simone Hettmer (D) https://orcid.org/0000-0003-1709-4448

Kornelius Kerl (D) https://orcid.org/0000-0002-5676-8102

Michael C. Frühwald (D) https://orcid.org/0000-0002-8237-1854

\section{REFERENCES}

1. Kieran MW, Roberts CW, Chi SN, et al. Absence of oncogenic canonical pathway mutations in aggressive pediatric rhabdoid tumors. Pediatr Blood Cancer. 2012;59:1155-1157.

2. Schneppenheim R, Fruhwald MC, Gesk S, et al. Germline nonsense mutation and somatic inactivation of SMARCA4/BRG1 in a family with rhabdoid tumor predisposition syndrome. Am J Hum Genet 2010;86:279-284.

3. Hasselblatt M, Nagel I, Oyen F, et al. SMARCA4-mutated atypical teratoid/rhabdoid tumors are associated with inherited germline alterations and poor prognosis. Acta Neuropathol. 2014;128:453-456.

4. Eaton KW, Tooke LS, Wainwright LM, Judkins AR, Biegel JA. Spectrum of SMARCB1/INI 1 mutations in familial and sporadic rhabdoid tumors. Pediatr Blood Cancer. 2011;56:7-15.

5. Fischer-Valuck BW, Chen I, Srivastava AJ, et al. Assessment of the treatment approach and survival outcomes in a modern cohort of patients with atypical teratoid rhabdoid tumors using the National Cancer Database. Cancer. 2017;123:682-687.

6. Schrey D, Carceller Lechon F, Malietzis G, et al. Multimodal therapy in children and adolescents with newly diagnosed atypical teratoid rhabdoid tumor: individual pooled data analysis and review of the literature. J Neurooncol. 2016;126:81-90.

7. Lafay-Cousin L, Hawkins C, Carret AS, et al. Central nervous system atypical teratoid rhabdoid tumours: the Canadian Paediatric Brain Tumour Consortium experience. Eur J Cancer. 2012;48:353-359.
8. Chi SN, Zimmerman MA, Yao X, et al. Intensive multimodality treatment for children with newly diagnosed CNS atypical teratoid rhabdoid tumor. J Clin Oncol. 2009;27:385-389.

9. Fruhwald MC, Biegel JA, Bourdeaut F, Roberts CW, Chi SN. Atypical teratoid/rhabdoid tumors-current concepts, advances in biology, and potential future therapies. Neuro Oncol. 2016;18:764-778.

10. Nemes K, Bens S, Kachanov D, et al. Clinical and genetic risk factors define two risk groups of extracranial malignant rhabdoid tumours (eMRT/RTK). Eur J Cancer. 2021;142:112-122.

11. Fruhwald MC, Hasselblatt $M$, Nemes $K$, et al. Age and DNAmethylation subgroup as potential independent risk factors for treatment stratification in children with atypical teratoid/rhabdoid tumors (ATRT). Neuro Oncol. 2020;22(7):1006-1017. https://doi.org/10.1093/ neuonc/noz244

12. Johann PD, Erkek S, Zapatka M, et al. Atypical teratoid/rhabdoid tumors are comprised of three epigenetic subgroups with distinct enhancer landscapes. Cancer Cell. 2016;29:379-393.

13. Torchia J, Golbourn B, Feng S, et al. Integrated (epi)-genomic analyses identify subgroup-specific therapeutic targets in CNS rhabdoid tumors. Cancer Cell. 2016;30:891-908.

14. Torchia J, Picard D, Lafay-Cousin L, et al. Molecular subgroups of atypical teratoid rhabdoid tumours in children: an integrated genomic and clinicopathological analysis. Lancet Oncol. 2015;16:569-582.

15. Chun HE, Lim EL, Heravi-Moussavi A, et al. Genome-wide profiles of extra-cranial malignant rhabdoid tumors reveal heterogeneity and dysregulated developmental pathways. Cancer Cell. 2016;29: 394-406.

16. Kantarjian H, Issa JP, Rosenfeld CS, et al. Decitabine improves patient outcomes in myelodysplastic syndromes: results of a phase III randomized study. Cancer. 2006;106:1794-1803.

17. Kantarjian HM, Thomas XG, Dmoszynska A, et al. Multicenter, randomized, open-label, phase III trial of decitabine versus patient choice, with physician advice, of either supportive care or low-dose cytarabine for the treatment of older patients with newly diagnosed acute myeloid leukemia. J Clin Oncol. 2012;30:2670-2677.

18. Gore L, Triche TJ, Farrar JE, et al. A multicenter, randomized study of decitabine as epigenetic priming with induction chemotherapy in children with AML. Clin Epigenetics. 2017;9:108.

19. Benton CB, Thomas DA, Yang $\mathrm{H}$, et al. Safety and clinical activity of 5-aza-2'-deoxycytidine (decitabine) with or without hyper-CVAD in relapsed/refractory acute lymphocytic leukaemia. $\mathrm{Br} J$ Haematol. 2014;167:356-365.

20. Phillips CL, Davies SM, McMasters R, et al. Low dose decitabine in very high risk relapsed or refractory acute myeloid leukaemia in children and young adults. Br J Haematol. 2013;161:406-410.

21. George RE, Lahti JM, Adamson PC, et al. Phase I study of decitabine with doxorubicin and cyclophosphamide in children with neuroblastoma and other solid tumors: a Children's Oncology Group study. Pediatr Blood Cancer. 2010;55:629-638.

22. Chabot GG, Rivard GE, Momparler RL. Plasma and cerebrospinal fluid pharmacokinetics of 5-Aza-2'-deoxycytidine in rabbits and dogs. Cancer Res. 1983;43:592-597.

23. Bartelheim K, Nemes K, Seeringer A, et al. Improved 6-year overall survival in AT/RT - results of the registry study Rhabdoid 2007. Cancer Med. 2016;5:1765-1775. https://doi.org/10.1093/neuonc/noz244

24. Worst BC, Van Tilburg CM, Balasubramanian GP, et al. Nextgeneration personalised medicine for high-risk paediatric cancer patients - The INFORM pilot study. Eur J Cancer. 2016;65:91-101.

25. Capper D, Jones DTW, Sill M, et al. DNA methylation-based classification of central nervous system tumours. Nature. 2018;555:469474.

26. Grill J, Geoerger B, Gesner L, et al. Phase II study of irinotecan in combination with temozolomide (TEMIRI) in children with recurrent or refractory medulloblastoma: a joint ITCC and SIOPE brain tumor study. Neuro Oncol. 2013;15:1236-1243. 
27. Nonnenmacher L, Westhoff MA, Fulda S, et al. RIST: a potent new combination therapy for glioblastoma. Int J Cancer. 2015;136:E173187.

28. Kerl K, Moreno N, Holsten T, et al. Arsenic trioxide inhibits tumor cell growth in malignant rhabdoid tumors in vitro and in vivo by targeting overexpressed Gli1. Int J Cancer. 2014;135:989-995.

29. Kieran MW, Turner CD, Rubin JB, et al. A feasibility trial of antiangiogenic (metronomic) chemotherapy in pediatric patients with recurrent or progressive cancer. J Pediatr Hematol Oncol. 2005;27:573581.

30. Athale UH, Duckworth J, Odame I, Barr R. Childhood atypical teratoid rhabdoid tumor of the central nervous system: a meta-analysis of observational studies. J Pediatr Hematol Oncol. 2009;31:651-663.

31. Venkataraman S, Alimova I, Tello T, et al. Targeting aurora kinase A enhances radiation sensitivity of atypical teratoid rhabdoid tumor cells. J Neurooncol. 2012;107:517-526.

32. Lee S, Cimica V, Ramachandra N, Zagzag D, Kalpana GV. Aurora A is a repressed effector target of the chromatin remodeling protein INI1/hSNF5 required for rhabdoid tumor cell survival. Cancer Res. 2011;71:3225-3235.

33. Alimova I, Birks DK, Harris PS, et al. Inhibition of EZH2 suppresses selfrenewal and induces radiation sensitivity in atypical rhabdoid teratoid tumor cells. Neuro Oncol. 2013;15:149-160.

34. Arnhold V, Oyen F, Schneppenheim R, et al. Long-term survival of an infant with an atypical teratoid/rhabdoid tumor following subtotal resection and low-cumulative dose chemotherapy: a case report. Childs Nerv Syst. 2016;32:1157-1161.

35. Hashizume R, Zhang A, Mueller S, et al. Inhibition of DNA damage repair by the CDK4/6 inhibitor palbociclib delays irradiated intracranial atypical teratoid rhabdoid tumor and glioblastoma xenograft regrowth. Neuro Oncol. 2016;18:1519-1528.

36. Nemes K, Fruhwald MC. Emerging therapeutic targets for the treatment of malignant rhabdoid tumors. Expert Opin Ther Targets. 2018;22(4):365-379. https://doi.org/10.1080/14728222.2018. 1451839

37. Wetmore C, Boyett J, Li S, et al. Alisertib is active as single agent in recurrent atypical teratoid rhabdoid tumors in 4 children. Neuro Oncol. 2015;17:882-888.

38. Mosse YP, Fox E, Teachey DT, et al. A phase II study of alisertib in children with recurrent/refractory solid tumors or leukemia: Children's Oncology Group phase I and pilot consortium (ADVL0921). Clin Cancer Res. 2019;25(11):3229-3238. https://doi.org/10.1158/10780432.CCR-18-2675

39. Gotti G, Biassoni V, Schiavello E, et al. A case of relapsing spinal atypical teratoid/rhabdoid tumor (AT/RT) responding to vinorelbine, cyclophosphamide, and celecoxib. Childs Nerv Syst. 2015;31:16211623.

40. Berland M, Padovani L, Rome A, Pech-Gourg G, Figarella-Branger D, Andre N. Sustained complete response to metronomic chemotherapy in a child with refractory atypical teratoid rhabdoid tumor: a case report. Front Pharmacol. 2017;8:792.

41. Geoerger B, Bourdeaut F, DuBois SG, et al. A phase I study of the CDK4/6 inhibitor ribociclib (LEE011) in pediatric patients with malignant rhabdoid tumors, neuroblastoma, and other solid tumors. Clin Cancer Res. 2017;23:2433-2441.

42. Rare tumors in kids may respond to tazemetostat. Cancer Discov. 2018;8: OF5.

43. Lin NU, Lee EQ, Aoyama $\mathrm{H}$, et al. Response assessment criteria for brain metastases: proposal from the RANO group. Lancet Oncol. 2015;16:e270-278.

44. Gerwing M, Herrmann K, Helfen A, et al. The beginning of the end for conventional RECIST - novel therapies require novel imaging approaches. Nat Rev Clin Oncol. 2019;16:442-458.
45. Seymour L, Bogaerts J, Perrone A, et al. iRECIST: guidelines for response criteria for use in trials testing immunotherapeutics. Lancet Oncol. 2017;18:e143.

46. Le Lay J, Jarraya H, Lebellec L, Penel N. irRECIST and iRECIST: the devil is in the details. Ann Oncol. 2017;28:1676-1678.

47. Glasser CL, Lee A, Eslin D, Marks L, Modak S, Glade Bender JL. Epigenetic combination therapy for children with secondary myelodysplastic syndrome (MDS)/acute myeloid leukemia (AML) and concurrent solid tumor relapse. J Pediatr Hematol Oncol. 2017;39:560564.

48. Kearns P, Zwaan CM, Reinhardt D, et al. Phase 1-2 safety, efficacy and pharmacokinetic study of decitabine in sequential administration with cytarabine in children with relapsed or refractory acute myeloid leukaemia. Br J Haematol. 2019;186(3):e7-e11.

49. Upadhyaya SA, Robinson GW, Onar-Thomas A, et al. Relevance of molecular groups in children with newly diagnosed atypical teratoid rhabdoid tumor: results from prospective St. Jude multi-institutional trials. Clin Cancer Res. 2021;27:2879-2889.

50. Lindblad KE, Goswami M, Hourigan CS, Oetjen KA. Immunological effects of hypomethylating agents. Expert Rev Hematol. 2017;10:745752.

51. Chiappinelli KB, Strissel PL, Desrichard A, et al. Inhibiting DNA methylation causes an interferon response in cancer via dsRNA including endogenous retroviruses. Cell. 2015;162:974-986.

52. Roulois D, Singhania R, et al. DNA-demethylating agents target colorectal cancer cells by inducing viral mimicry by endogenous transcripts. Cell. 2015;162:961-973.

53. Nie J, Wang C, Liu Y, et al. Addition of low-dose decitabine to anti-pd-1 antibody camrelizumab in relapsed/refractory classical Hodgkin lymphoma. J Clin Oncol. 2019:JCO1802151.

54. Nie J, Zhang Y, Li X, Chen M, Liu C, Han W. DNA demethylating agent decitabine broadens the peripheral T cell receptor repertoire. Oncotarget. 2016;7:37882-37892.

55. Falchi L, Sawas A, Deng C, et al. High rate of complete responses to immune checkpoint inhibitors in patients with relapsed or refractory Hodgkin lymphoma previously exposed to epigenetic therapy. J Hematol Oncol. 2016;9:132.

56. Krishnadas DK, Shapiro T, Lucas K. Complete remission following decitabine/dendritic cell vaccine for relapsed neuroblastoma. Pediatrics. 2013;131:e336-341.

57. Krishnadas DK, Shusterman S, Bai F, et al. A phase I trial combining decitabine/dendritic cell vaccine targeting MAGE-A1, MAGE-A3 and NY-ESO-1 for children with relapsed or therapy-refractory neuroblastoma and sarcoma. Cancer Immunol Immunother. 2015;64:12511260.

58. Oronsky BT, Oronsky AL, Lybeck M, et al. Episensitization: defying time's. Arrow Front Oncol. 2015;5:134.

59. Chen M, Nie J, Liu Y, et al. Phase Ib/II study of safety and efficacy of low-dose decitabine-primed chemoimmunotherapy in patients with drug-resistant relapsed/refractory alimentary tract cancer. Int J Cancer. 2018;143:1530-1540.

60. Appleton K, Mackay HJ, Judson I, et al. Phase I and pharmacodynamic trial of the DNA methyltransferase inhibitor decitabine and carboplatin in solid tumors. J Clin Oncol. 2007;25:46034609.

61. Plumb JA, Strathdee G, Sludden J, Kaye SB, Brown R. Reversal of drug resistance in human tumor xenografts by 2'-deoxy-5-azacytidineinduced demethylation of the hMLH1 gene promoter. Cancer Res. 2000;60:6039-6044.

62. Benson EA, Skaar TC, Liu Y, Nephew KP, Matei D. Carboplatin with decitabine therapy, in recurrent platinum resistant ovarian cancer, alters circulating miRNAs concentrations: a pilot study. PLoS One. 2015;10:e0141279. 
63. Carter CA, Oronsky BT, Caroen SZ, et al. Partial response to platinum doublets in refractory EGFR-positive non-small cell lung cancer patients after RRx-001: evidence of episensitization. Case Rep Oncol. 2016;9:62-67.

64. Fang F, Zuo Q, Pilrose J, et al. Decitabine reactivated pathways in platinum resistant ovarian cancer. Oncotarget. 2014;5:3579-3589.

65. Matei D, Fang F, Shen C, et al. Epigenetic resensitization to platinum in ovarian cancer. Cancer Res. 2012;72:2197-2205.

66. Qin T, Si J, Raynal NJ, et al. Epigenetic synergy between decitabine and platinum derivatives. Clin Epigenetics. 2015;7:97.

67. Melcher V, Graf M, Interlandi M, et al. Macrophage-tumor cell interaction promotes ATRT progression and chemoresistance. Acta Neuropathol. 2020;139:913-936.

68. Chun HE, Johann PD, Milne $K$, et al. Identification and analyses of extra-cranial and cranial rhabdoid tumor molecular subgroups reveal tumors with cytotoxic T cell infiltration. Cell Rep. 2019;29:2338-2354 e2337.

69. Leruste A, Tosello J, Ramos RN, et al. Clonally expanded T cells reveal immunogenicity of rhabdoid tumors. Cancer Cell. 2019;36(6):597612.e8. https://doi.org/10.1016/j.ccell.2019.10.008

70. Lindblad KE, Thompson J, Gui G, et al. Pembrolizumab and decitabine for refractory or relapsed acute myeloid leukemia. Blood. 2018;132
(Supplement 1):1437-1437. https://doi.org/10.1182/blood-2018-99115097

71. Stresemann C, Lyko F. Modes of action of the DNA methyltransferase inhibitors azacytidine and decitabine. Int J Cancer. 2008;123:8-13.

72. Ho B, Johann PD, Grabovska Y, et al. Molecular subgrouping of atypical teratoid/rhabdoid tumors-a reinvestigation and current consensus. Neuro Oncol. 2020;22:613-624.

\section{SUPPORTING INFORMATION}

Additional supporting information may be found online in the Supporting Information section at the end of the article.

How to cite this article: Steinbügl M, Nemes K, Johann P, et al. Clinical evidence for a biological effect of epigenetically active decitabine in relapsed or progressive rhabdoid tumors. Pediatr Blood Cancer. 2021;68:e29267.

https://doi.org/10.1002/pbc.29267 